Casos Clínicos

Arch. Esp. Urol. 2010; 63 (7): 550-554

\section{ADENOCARCINOMA DE URACO CON METÁSTASIS CEREBRALES TARDIAS}

\author{
Celestino Gómez Rebollo, Antonio Javier Márquez \\ Moreno, Francisco Antuña Calle', Lidia Pérez Villa², \\ Juan José Sánchez Carrillo², Emilio Julve Villalta', \\ Alfredo Blanes Berenguel² y Mercedes Acebal Blanco.
}

Servicios de Radiología, Urología' y Anatomía Patológica². Hospital Clínico de Málaga. Málaga. España.

Resumen.- OBJETIVO: Describir un caso de adenocarcinoma uracal con metástasis cerebrales tardías en un paciente de 61 años que consultó por molestias abdominales y hematuria de 6 meses de evolución.

MÉTODO: Con la sospecha clínica de tumoración vesical se realizaron pruebas diagnósticas /citología seriada de orina, cistoscopia, ecografía abdominal y TAC abdominopélvico). Se indicó tratamiento quirúrgico.

RESULTADOS: La citología de orina fué negativa. En la cistoscopia se observó una lesión infiltrante en la cúpula vesical. En la ecografía y la TAC se apreció una lesión redondeada de $5 \mathrm{~cm}$, con densidad intermedia, ecos internos y

\section{CORRESPONDENCIA}

Antonio Javier Márquez Moreno Teseo, 5 3-N 29010 Málaga (España). ajmmrx@hotmail.es Aceptado para publicar: 28 de octubre 2009. calcificaciones en la línea media supravesical anterior, que infiltraba la vejiga. El estudio de extensión no mostró hallazgos. Se realizó cistectomía parcial y linfadenectomía. El diagnóstico histopatológico fué adenocarcinoma uracal mucosecretor. Tras 5 años libre de enfermedad el paciente desarrolló metástasis pulmonar y cerebrales.

CONCLUSIONES: El adenocarcinoma de uraco es un tumor que debe distinguirse del adenocarcinoma primario vesical. El tipo mucosecretor puede asociar calcificaciones detectables en las pruebas de imagen que deben hacer sospechar el diagnóstico. La presencia de metástasis tardías (tras 5 años libre de enfermedad) y sin signos de recidiva local es un hecho clinicopatológico infrecuente.

Palabras clave: Adenocarcinoma. Uraco. Vejiga. TAC. Ecografía.

Summary.- OBJECTIVE: To describe a case of urachal adenocarcinoma with late brain metastases in a sixtyone year old man who presented abdominal discomfort and hematuria during six months.

METHODS: The clinical suspicion was bladder tumor and diagnostic studies were performed /urinary cyłology, cystoscopy, abdominal ultrasound and abdominopelvic CT scan). Surgical treatment was performed.

RESULTS: Negative urinary cytology. Cystoscopy showed a lesion with infiltration of the bladder dome. Ultrasound and CT scan showed a five centimeter rounded lesion, with intermediate density, internal echoes and calcifications on the anterior supravesical middle line, that infiltrated the bladder. The extension study had not findings. Partial cystectomy and lymphadenectomy were performed. The histopathologic diagnosis was mucin-secreting urachal adenocarcinoma.

After five years without disease the patient suffered lung and brain metastases.

CONCLUSIONS: Urachal adenocarcinoma is a tumor which must be distinguished of primary bladder adenocarcinoma. The mucing-secreting adenocarcinoma can be associated with calcifications that can be demostrated on imaging studies. Late metastases without signs of local recurrence (after five years without disease) are an infrequent clinical-pathologic finding.

Keywords: Adenocarcinoma. Urachus. Bladder. CT. Sonography.

\section{INTRODUCCIÓN}

El carcinoma de uraco es un tumor extremadamente raro, con una incidencia de aproximadamente 1/5.000.000 de habitantes (menos del 0,001 de todos los tumores 
de vejiga) diagnosticándose habitualmente de forma tardía y asociando un mal pronóstico, apreciándose, generalmente, recidiva local y enfermedad metastásica en los 24 meses siguientes al diagnóstico $(1,2)$. A continuación presentamos un caso de adenocarcinoma de uraco infiltrante con metástasis cerebrales relativamente tardías (después de un periodo libre de enfermedad de 5 años y sin evidencia clínica de recidiva local) en relación con los casos descritos en la literatura.

\section{CASO CLÍNICO}

Paciente varón de 61 años, en tratamiento por psicosis esquizofrénica, sin otros antecedentes médico-quirúrgicos de interés, que consultó por molestias abdominales inespecíficas y hematuria macroscópica de 6 meses de evolución. En la analítica destacaba discreta anemia siendo los parámetros bioquímicos normales. En la exploración física no se encontraron hallazgos de interés abdómino-pélvicos, con exploración urológica sugestiva de hiperplasia prostática, sin otros hallazgos. PSA normal. Citología de orina negativa para células malignas. En las pruebas diagnósticas realizadas se apreció: cistoscopia (lesión única en cúpula vesical, sólida y de aspecto infiltrante; ecografía y TAC (lesión nodular redondeada, a nivel de la línea media supravesical, de unos $5 \mathrm{~cm}$ de diámetro sin plano de clivaje con la cúpula vesical, con contenido quístico denso y calcificaciones) (Figura 1 y $2 A$ ). No se apreció en el estudio de extensión por TC afectación torácica ni abdominopélvica. No adenopatías. TAC craneal normal. El paciente se intervino, realizándose una cistectomía parcial, cursando el postoperatorio y los controles oncológicos posteriores sin incidencias. El estudio histopatológico de la pieza quirúrgica mostró tejido uracal con presencia de un adenocarcinoma de tipo mucosecretor (coloide) (Figura 3) que infiltraba la pared vesical, compatible con neoplasia uracal infiltrante (estadio IIIA). Aproximadamente a los 5 años del diagnóstico se detectó primero una masa pulmonar redondeada que creció en posteriores controles rechazando el paciente y familiares el estudio por PAAF/ biopsia, y posteriormente metástasis cerebrales múltiples, no existiendo ninguna evidencia de otra enfermedad neoplásica clínica, radiológicamente ni en estudios de marcadores tumorales (Figura 2B).

\section{DISCUSIÓN}

El uraco, estructura anatómica fetal tubular que conecta la vejiga primitiva con el alantoides en el ombligo, involuciona aproximadamente a los 4-5 meses de gestación, persistiendo un vestigio en forma de delgado cordón fibroso, de longitud variable $(3$ y $10 \mathrm{~cm})$ situado en el tejido conectivo del espacio de Retzius $(2,3)$. La patología del uraco se puede resumir en alteraciones congénitas (uraco permeable) o adquiridas (fístula uracal) que cursan con comunicación completa vésico-umbilical y emisión de orina por ombligo; o aquellas alteraciones con comunicación incompleta, adquiridas: divertículo vesicouracal, quistes de uraco (uracovesicales o uracoumbilicales), sinus alternante (quiste con fistulización periódica) y tumores benignos (adenomas) o malignos (adenocarcinomas, carcinomas uroteliales y escamosos), así como cualquier sobreinfección o absceso complicando las patologías anteriores. También han sido descritos otros procesos más raros como malacoplaquia y xantogranulomas (Tabla I. Patología del uraco) (1-4).

El adenocarcinoma constituye el tumor maligno más habitual del uraco, siendo la variante mucosecretora lo
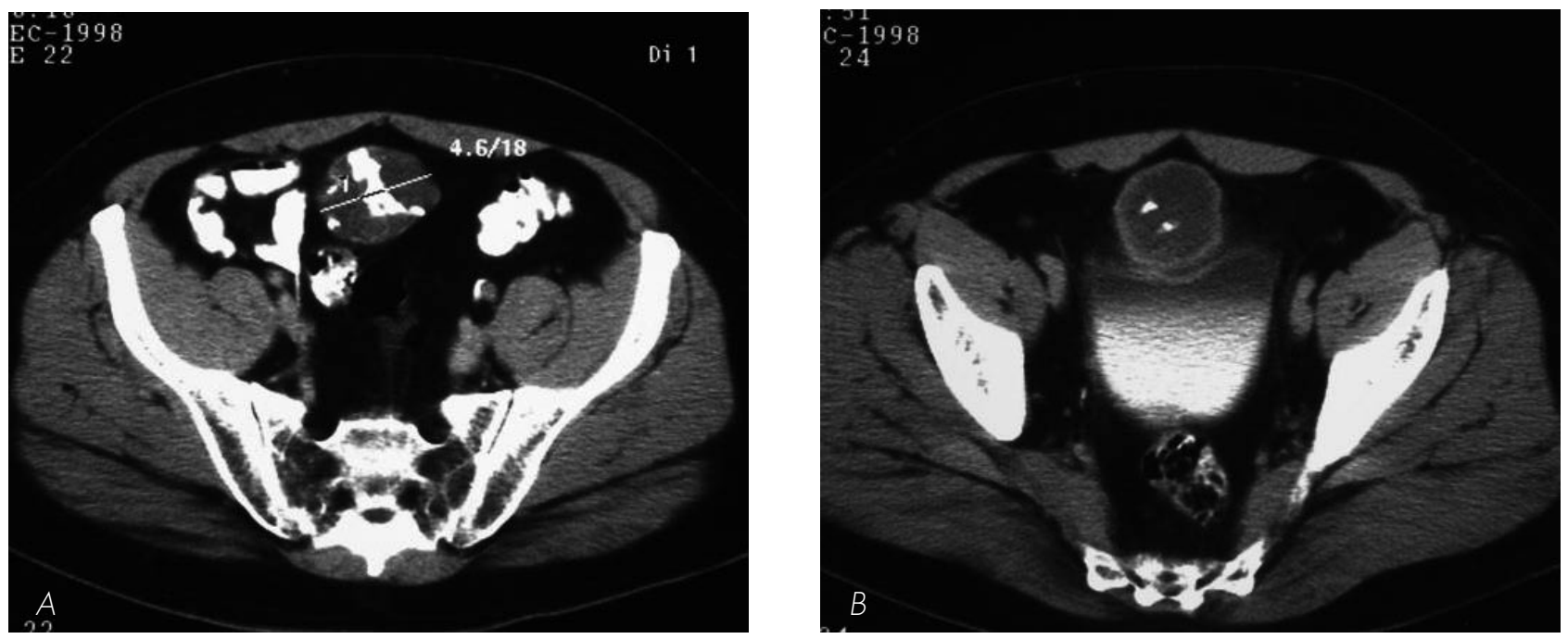

FIGURA 1. TAC abdominal. A y B. Lesión redondeada con densidad intermedia y calcificaciones en la línea media supravesical anterior. 

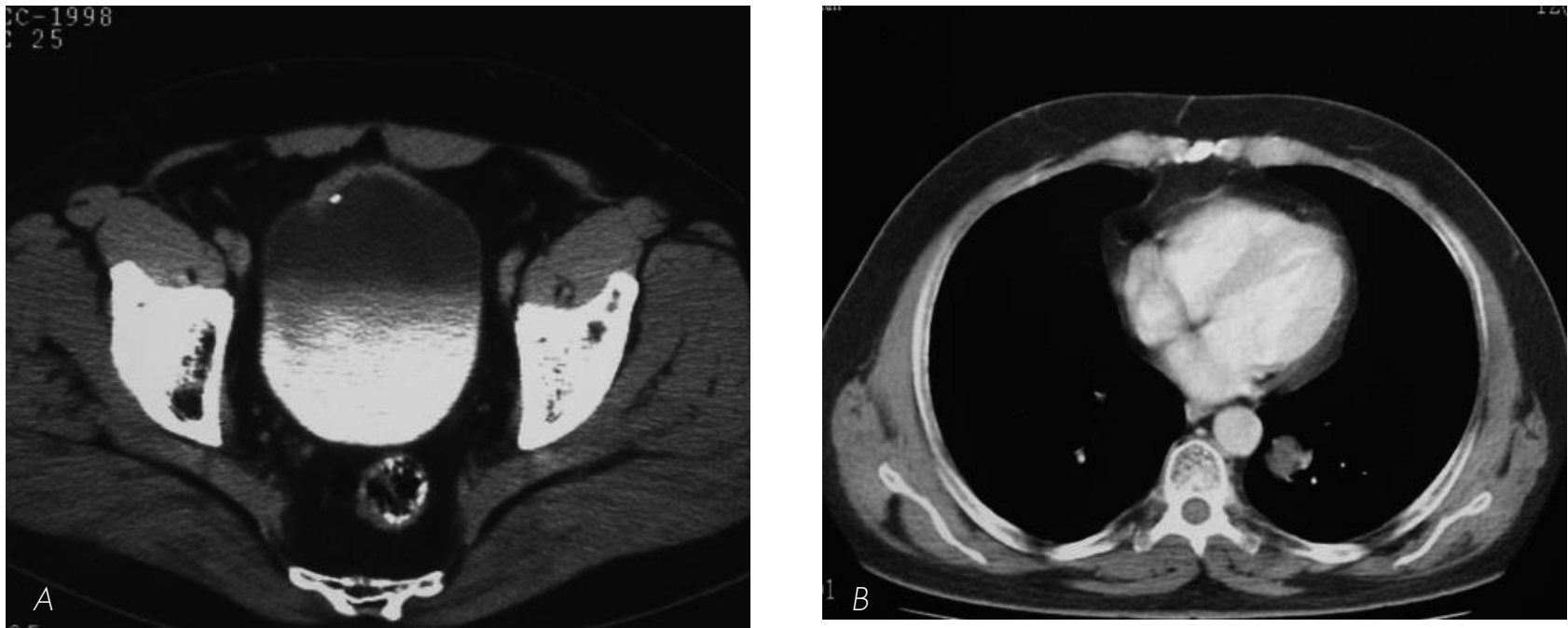

FIGURA 2. A. TC abdominal. Invasión (realce tumoral y calcificación) de la pared vesical. B. TAC torácico. Metástasis pulmonar.

coloide) el tipo histológico predominante $(70-90 \%$ de los casos). Afecta más frecuentemente a varones $\left(5^{\mathrm{a}}-7^{\mathrm{a}}\right.$ década de la vida), con una relación de 4:1 (4).

Clínicamente el síntoma más frecuente es la hematuria (70\% de los casos), que se produce cuando el tumor invade la pared vesical y traduce por lo tanto un estadío avanzado de enfermedad. Puede ocasionar síntomas más inespecíficos como molestias hipogástricas y

\section{TABLA I. PATOLOGÍA DEL URACO.}

$$
\begin{aligned}
& \text { - Uraco permeable congénito } \\
& \text { - Fístula uracal adquirida } \\
& \text { - Divertículo uracovesical } \\
& \text { - Quiste de uraco (uracoumbilical o uracovesical) } \\
& \text { - Sinus alternante (quiste + fístula periódica) } \\
& \text { - Tumores benignos (adenomas) } \\
& \text { - Tumores malignos: } \\
& \text { - Adenocarcinomas (coloide -el más frecuente-, } \\
& \text { tubular, papilar, en anillo de sello) } \\
& \text { - Carcinomas uroteliales } \\
& \text { - Carcinoma epidermoide } \\
& \text { - Infecciones y abscesos }
\end{aligned}
$$

- Otras patologías: malacoplaquia, xantogranulomas disuria, así como otros más raros y sugerentes como descarga mucoide vesical o umbilical. En algún caso aislado se ha apreciado diseminación tumoral en forma de pseudomixoma peritoneal, con distensión abdominal y clínica infecciosa secundaria. El estadiaje del tumor se establece mediante la clasificación de Sheldon (Tabla II. Estadiaje del carcinoma de uraco) $(5,6)$.

El diagnóstico se realiza de forma similar al de otro tumor vesical, siendo la hematuria el síntoma de sospecha y detectándose la lesión mediante cistoscopia (masa tumoral infiltrante o a veces se han descrito como áreas edematosas únicas en cúpula vesical) y las pruebas radiológicas habituales. En este sentido la placa simple de abdomen generalmente es normal, aunque a veces demuestra calcificaciones (asociadas al componente mucosecretor del tumor). En la urografía intravenosa se puede ver el defecto de repleción en la cúpula vesical y el signo de la fuga de contraste en la placa postmiccional. En la ecografía, TAC y RM, además de la extensión, la infiltración de la pared vesical y de otras estructuras abdominales y la afectación ganglionar, se aprecia el crecimiento predominantemente extravesical de la lesión con una densidad intermedia y ecos internos debido al contenido mucinoso, la porción sólida de la neoplasia con captación de contrate y las calcificaciones $38 \%$ de los casos en TC). En RM el tumor tiene una señal heterogénea con hiperintensidad en secuencias $T 2$, vacíos de señal por las calcificaciones y realce tras administración de gadolinio. Las pruebas radiológicas también ayudan a diferenciar el carcinoma uracal (crecimiento predominantemente extravesical y restos de uraco) del carcinoma de la cúpula vesical y descartan otras neoplasias primarias reduciendo la posibilidad de metástasis vesicales. La confirmación es histopatológica. Dado el orígen embriológico endodérmico del uraco, relacionado con el intestino primitivo, estos tumores pueden elevar 
marcadores tumorales como CEA, Ca 19.9 y alfa-fetoproteína (5-8).

El principal diagnóstico diferencial se realiza con el adenocarcinoma primario vesical, siguiendo los criterios de Wheeler y Mostofi:

a) tumor localizado en la cúpula vesical con desarrollo extravesical o intramural,

b) mucosa vesical adyacente normal con ausencia de áreas de cistitis quística o glandular,

c) no evidencia de tumor primario en otra localización,

d) existencia de restos uracales con tumor o crecimiento del tumor hacia la vejiga con extensión al espacio de Retzius, pared anterior del abdomen o al ombligo (9).

El tratamiento es quirúrgico, pudiendo realizarse cistectomía parcial en bloque con extirpación de todos los tejidos afectados y con un margen de seguridad, o cistectomía radical con derivación urinaria. En ambos casos se completa con linfadenectomía. El tratamiento adyuvante con radioterapia y quimioterapia no ha mostrado clásicamente resultados significativos, si bien las terapias actuales con cisplatino parecen más esperanzadoras. Actualmente la supervivencia media a los 5 años es de un $43-50 \%$. El pronóstico no es bueno ya que generalmente se diagnostican en un estadio avanzado, siendo frecuentes las recidivas locales $(30 \%)$ y las metástasis a distancia $137 \%$ y generalmente asociada a recidiva local) en los siguientes 24 meses posteriores al diagnóstico. Otros factores relacionados con la supervivencia son el tipo de cirugía realizado y el estado de los márgenes quirúrgicos, la afectación de ganglios linfáticos y el subtipo y la diferenciación tumoral. Las metástasis afectan a hueso, médula, pulmón, hígado,

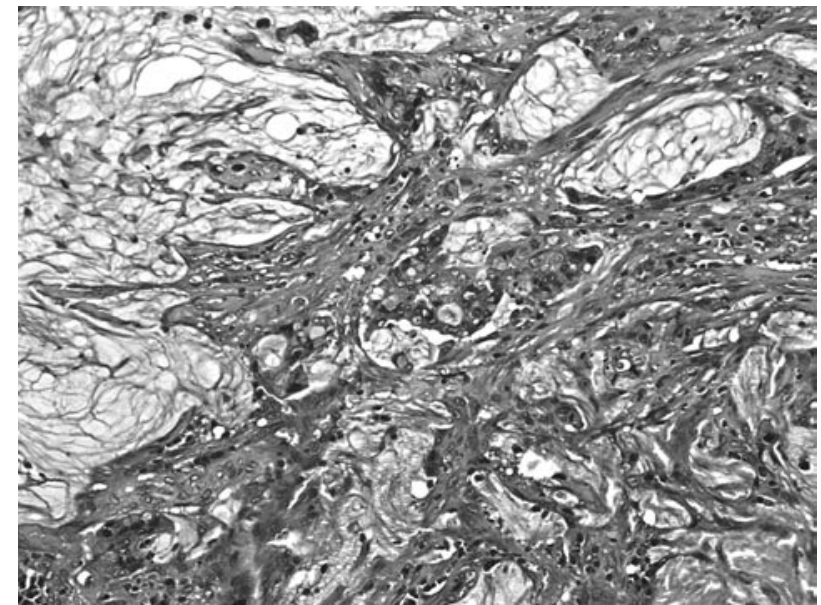

\section{FIGURA 3. Histopatología. Glándulas tumorales con} abundante material mucoide.

diseminación peritoneal, ganglios linfáticos regionales y más raramente a cerebro, habiéndose descrito casos rarísimos de metástasis en ovarios y en maxilar. En nuestro caso se apreció, tras un periodo libre de enfermedad superior al normal (24 meses vs 5 años), metástasis pulmonares y cerebrales (relativamente raras en este tumor), sin evidencia alguna de recidiva local (8-10).

\section{CONCLUSIONES}

El adenocarcinoma de uraco presenta características clínico-radiológicas (crecimiento extravesical, calcificaciones detectables en radiología simple o TC, "signo de la fuga de contraste", etc) que ayudan a diferenciarlo de los tumores vesicales primarios. La presencia de me-

TABLA II. ESTADIAJE DE SHELDON DEL CARCINOMA URACAL.

ESTADIO I: Tumor limitado a la mucosa vesical uracal

ESTADIO II: Tumor que invade submucosa o muscular pero limitado al uraco

ESTADIO III: Extensión fuera del uraco

III A: Invade vejiga

III B: Invade la pared abdominal

III C: Invasión peritoneal

III D: Invade vísceras locales

ESTADIO IV: Metástasis a distancia 
tástasis tardías (tras 5 años libre de enfermedad) y $\sin$ signos de recidiva local es un hecho clinicopatológico infrecuente. El tratamiento es quirúrgico y el pronóstico malo.

\section{BIBLIOGRAFÍA Y LECTURAS RECOMENDADAS (*lectura de interés $y$ ** lectura fundamental)}

*1. Martínez-Cornelio A, Flores-López D, Flores-Ojeda $\mathrm{R}$ et al. Experiencia quirúrgica en el adenocarcinoma de uraco. Cir Ciruj, 2009; 77: 33-8.

**2. Sheldon CA, Clayman RV, González R et al. Malignant urachal lesions. J Urol, 1984; 131: 1-8.

*3. Mostofi FK, Thomson RV, Dean AL. Mucus adenocarcinoma involving the urinary bladder. Cancer 1995; 8: 741-58.

*4. Ojea A, Núñez A, Domínguez F et al. Adenocarcinoma mucinoso de uraco. Actas Urol Esp [on line] 2003, 27: 142-6.

**5. Gutiérrez E, Espiga FJ, Arroyo JL et al. Bladder adenocarcinoma. Presentation of 3 new cases. Arch Esp Urol, 1991; 44: 141-3.

6. Donate Moreno MJ, Giménez Bachs JM, Salinas Sánchez AS et al. Patología del uraco: revisión de conjunto y presentación de tres casos. Actas Urol Esp, 2005; 29: 332-6.

**7. Soto Delgado M, Pedrero Márquez G, Varo Solís C et al. Adenocarcinoma Mucinoso de Uraco y Pseudomixoma Peritoneal. Actas Urol Esp, 2006; 30: 222-6.

**8. Gopalan A, Sharp DS, Fine SW et al. Urachal carcinoma: a clinicopathologic analysis of 24 cases with outcome correlation. Am J Surg Pathol. 2009; 33: 659-68.

**9. Nimmonrat A, Na-ChiangMai W, Muttarak M. Urachal abnormalities: clinical and imaging features. Singapore Med J. 2008; 49: 930-5.

**10. Paras FA Jr, Maclennan GT. Urachal adenocarcinoma. J Urol. 2008; 180:720. 